\title{
Theory of Filipino Substance Use Recovery (SUR): A Grounded Theory Approach
}

\author{
Karen P. Sol ${ }^{1}$ and Jemerson N. Dominguez ${ }^{2 *}$ \\ ${ }^{1)}$ Department of Health-Treatment and Rehabilitation Center, Philippines \\ ${ }^{2}$ De La Salle University - Dasmariñas, Philippines \\ *jndominguez@dlsud.edu.ph
}

\begin{abstract}
Treatment for Substance Use Disorder (SUD) is often theory-based however, recovery has not been guided by a theory. This grounded theory study intended to identify how an individual maintains recovery from SUD. Twenty (20) recovering persons as the participants in a one-on-one in-depth interview employing Charmaz's grounded theory methodology of analyzing data, a theory was developed. The theory of Filipino Substance Use Recovery (SUR) generated five theoretical phases: Exhaustion, Recognition, Acceptance, Involvement, and Maintenance. Under the Maintenance phase, components in maintaining a lifelong recovery were also identified such as autonomy, maturity, support system, positive feelings, and restitution. Furthermore, it recognized two recovery barriers (internal and external barriers) thus explained the phenomena of substance use relapse. In conclusion, the theoretical foundation of Filipino SUR could help improve the current treatment and management of SUD in the Philippines. Findings could be utilized in developing programs grounded and contextualized in the Philippine setting to address issues in substance use prevention, harm reduction, intervention, and recovery.
\end{abstract}

Keywords: Substance Use Disorder (SUD), recovery, relapse, grounded theory

\section{INTRODUCTION}

Substance Use Disorder (SUD) is considered a brain disease characterized by an irresistible impulse to use and seek drugs regardless of the negative consequences with the chronic and relapsing disorder that affects a significant portion of people in the community (Goldstein \& Volkow, 2011). This involves behavioral abnormality for vulnerable individuals that have been repeatedly exposed to drug abuse. Potentially life-long functional changes to the brain circuits associated with self-control, rewards, and stress may remain for a long time even despite the cessation of drug use (Moeller \& Goldstein, 2014). As one of the primary public concerns across generations is the use of illicit drugs. This affects not only the individual but also their family like successive fights of each family member under drug use, and the violence and crime to its community.
Substance use has no homogenous case, they are extremely heterogeneous. The individual has his reasons for abusing the drugs, childhood experiences and environmental attribution before taking the drugs, the effects, the cravings, the withdrawal syndrome, extent of dependency, and duration of recovery. The individual's journey to substance use recovery (SUR) is never identical by culture, each person has their unique path. For some, they were able to stop substance use and continue to recover, others had relapsed, and some do not start recovering at all. Winters, Botzet, and Fahnhorst (2011) explained that most substance users will return to treatment often more than once in their lifetime. About a quarter of a billion people who used drugs and around 29.5 million people of $0.6 \%$ of the global adult population were also engaged in problematic use, suffered from mental disorders 
alongside medical, social, psychological, and legal problems. The reported drug-related deaths and mortality rates in Asia consist of 32.518 out of 452.043 deaths worldwide mostly due to fatal substance use overdose excluding other co-occurring psychiatric and other problems. The most common onset for substance use problems is during early adulthood yet the stability of the diagnosis cannot be given at that point.

Data from the Nationwide Survey on the Nature and Extent of Drug Abuse in the Philippines, the problem of substance use in the country remains to be one of the major social concerns for the longest time since consumption of addictive substances (e.g., beverages with intoxicating capacity, chewable herbs, etc.) were not socially disapproved before the $19^{\text {th }}$ century. Statistics revealed an estimated 1.8 million people who are currently drug users. Equally, the Dangerous Drugs Board reported that the mean age of substance users in the Philippines was 32 years old, single, with a ratio of 9:1 (male to female), unemployed, and mostly high school level. Being a mono-drug-user means been using for the duration of 6 years prior to the survey, majority lives in the urban areas, and even more alarming is that drug abusers are getting younger. Over the last decade, numbers revealed a drift towards a decreasing age ranged from 40-55 years old during the '50s, the average age of 25 years old on the ' 80 s, and more recent data indicate that initiation to drug use starts at the young age of 8-9.

Relapse to substance use carries a heavy cost in terms of safety and public health and poses great harm to the person using the drugs, to their family and community. Despite that, there is a considerable reduction in substance use after undergoing treatment, it is often difficult to maintain thus it remains to be one of the greatest challenges in treatment and management of addictive behaviors (Whitesell, Bachand, Peel, \& Brown, 2013). Researchers and clinicians in the field acknowledged SUD as chronic thus militate treatment and abstinent efforts of the individual (Golestan et al., 2010).

Additionally, the Philippine Dangerous Drugs Board (PDDB) year-end survey revealed that $18 \%$ of the patients who underwent drug rehabilitation treatment had relapsed and were readmitted to a rehabilitation facility. The Philippine relapse rate from 2009 to 2016 ranged from $15-18 \%$ of the total admission in all the residential-based facilities nationwide while recovery rates remain unidentified.

Laudet (2013) argued that findings from most studies focused on starting SUR only, while the challenges and processes involved in attaining and supporting long-term recovery were overlooked. Further, they also emphasized that recovery is more than not taking drugs or alcohol in an unchanged life, hence it is important to look beyond stable recovery from substance use and broaden the investigative scope in the field. Meanwhile, recovery is coined as a process wherein an individual improves their health and wellness, live a self-directed life to reach their full potential and eventual change (SAMHSA, 2018); need to meet the dimensions like substance use disorder remission, global health enhancement in the physical, emotional, relational, occupational, and spiritual, and positive community inclusion (Whitesell et al., 2013); and embraces the recognition of the need for change and transformations, understanding different cultural dimensions, and merging hope and gratitude (Sheedy \& Whitter, 2013). Thus, recovery is generally viewed as a process that does not represent a cure but a state of being and becoming that involves addressing discrimination, stigma, and shame. It requires an individual to take personal accountability and responsibility for their recovery in collaboration with their families, friends, and professionals from the addiction field.

Hser, Evans, Grella, Ling, and Anglin (2015) revealed that treatment and interventions for SUD have been spread worldwide and they are effective, however, short-lived. Those who achieved a more stable recovery had cycled through several treatments before they were able to prolong the adverse consequences of substance abuse. The National Institute on Drug Abuse (NIDA) confirms that brain scans have shown that the individual's brain improves when terminated from prolonged drug use (NIDA, 2017). Long-term studies on SUD based on 
cohort treatments revealed that the problem of relapse has been present for decades and manifests a high risk of death (Hser et al, 2015). While Scott and Dennis (2012) supported that cumulative time of abstinence is a strong predictor of future recovery, however, treatment can only predict recovery but not substance use maintenance. Whereas McQuaid, Malik, Moussouni, Baydack, Stargardter, and Morrisey (2017) identified stigma and discrimination as a hindrance to maintaining SUR. This is characterized by being looked down, judged, shunned, or excluded by friends, family, and society in general. Abstinence from substance use depends heavily on the individuals' treatment completion. To be in remission, an individual must have a year of sobriety with no slips and manifestations of drug-related behaviors (Kleber et al, 2011). Scott, Dennis, Laudet, Funk, and Simeone (2011) mentioned the major predictor of abstinence is not treatment, but the degree of participation in a self-help group after treatment. Likely, individuals with higher substance use severity are not likely to shift from addiction to SUR when environmental obstacles are present which can lead to the possibility of relapse. Relapse is a phenomenon of the return to drug-related thoughts, emotions, and behaviors after a significant period of abstinence. Researchers have focused on examining the process of substance use relapse wherein adults and adolescents tend to use immediately after treatment for drug problems (Witkiewitz, 2011). Relapse is a very common phenomenon after treatment for drug addiction. Studies conducted in Asian countries revealed that substance use relapse could include depression, family disruption, and heavy use of the substance that might also lead to suicide, theft, murder, and other dreadful crimes.

Literature acknowledges the remarkable roles of emotional states in the relapse process like negative experiences such as emptiness, inadequacies, and frustration alongside other unpleasant or aversive emotions including loneliness, hopelessness, personal loss, and grief, have been cited in the process of relapse (Fitzgerald \& Puttler, 2018); confirming that failure to attain a specific goal, have led to relapse among recovering people due to frustrations which they tend to ease with the help of the effects of the illicit drugs.

As a research gap, the treatment for SUD is often theory-based however, the remission and recovery have not been guided by a theory. Furthermore, most researches do not focus on broader domains of addiction but have focused only on substance use outcomes. Despite the recent thrust of the people in the field of substance use and the historical surge of interest in recovery, related researches and analyses have been so limited. Efforts are directed towards determining the most effective treatment and providing measurements in the current continuum of systems addressing addiction as a chronic disease. Established cessation and recovery have received only a little attention in the field of research, thus the fact that despite understanding why people use drugs and interventions have been made to help them, there is still a deficiency for rich empirical analysis about recovery from substance use (Hser et al, 2015). Equally, the literature review revealed that there are no local studies conducted in the Philippines that explore the process of recovery in an individual with SUD. In addition, the government is currently utilizing varieties of programs and implementing treatment modalities for SUD management and rehabilitation such as the Therapeutic Community, 12-Steps, Matrix Model, etc. that are essentially rooted from foreign countries employing practices and principles that are not contextualized in the Philippine setting and among Filipino subjects. Thus, the lack of theoretical underpinning in the field of SUR contributes to the unresolved issue of substance use relapse and recidivism in the country. Therefore, this study aimed to develop a theoretical understanding and framework that would contribute to the modification of the current SUD treatment and management into a more recovery-oriented approach, grounded and well-suited in the Philippine setting. The present study aimed to identify the phases that a Filipino individual with SUD undergoes in the process of recovery. 


\section{METHOD}

The study employed a grounded theory approach which is intended to generate and develop theories following a systematic yet flexible process for collecting and analyzing qualitative data. to create theories grounded and obtained from the data themselves (Charmaz, 2006). In constructivist grounded theory research, the researcher's presence is strongly recognized as neither neutral nor undesirable in the research output, anchored to a key element in the topic selection, research preparation, collection of data, and analysis (Ramalho, Adams, Huggard, \& Hoare, 2015).

Twenty Filipinos recovering from SUD and have finished a treatment program in an inpatient/ residential rehabilitation facility met the criteria: (1) recovering to SUD for more than five years during this present study since this is the average span of stay in most facilities, and (2) has no co-occurring disorder. All are male, with ages ranging from 2863 years old, most of them were married and were college undergraduates. 11 participants have been recovering from SUD for five years now, while two have been recovering for seven years, three have been maintaining sobriety for 10 years, and the others were able to sustain their recovery for 13 and 18 years. The participant who was able to recover for the longest time reported maintaining his recovery for 30 years now. Moreover, most of the participants' drug of choice was methamphetamine. Mostly work in a drug treatment and rehabilitation center as Program Staff while some are Administrative Staff. Three of them ventured into business, driving, and cooking while the minority remains unemployed.

To account for the experiences of Filipino individuals recovering from SUD, an in-depth interview was conducted into two parts; first was the gathering of the demographic profile of the participants (including age, civil status, educational attainment, employment status, number of years in recovery, number of times rehabilitated or admitted in a facility, and drug of choice), followed by the one-on-one in-depth-interview that aimed to identify the process on how the participants started their journey to recovery, and how they were able to maintain it. The questions were validated by the experts in the addiction field credentialed as International Certified Addiction Professional Level II. The instrument was also pilot tested to five individuals recovering from substance use to ensure the appropriateness of questions to the target populations.

The study was conducted in the Cavite, Laguna, Batangas, Rizal, and Quezon (CALABARZON) Region where most of the participants are currently residing. The Ethical Clearance (DLSU-DERC2018-083T2) was successfully obtained from an Institutional Research Review Board. Permission was sought to different rehabilitation centers that treat individuals with SUD.

To obtain the data, informed consent signifying their voluntary participation was secured including assent, confidentiality, and data sharing. Coding was designated in the careful treatment of information in the data analysis to ensure anonymity. Afterward, gathered information will be destroyed. They were given the right to acquire a copy of the results of the study. The data were gathered from three different rehabilitation centers at the time that was convenient for the participants. Debriefing sessions were also conducted after every interview to guarantee that the data gathered did not cause any distress to the participants and they are protected from any physical or mental harm.

For data analysis, the researchers analyzed the verbatim transcriptions of the recorded interviews. The coding procedure is a pivotal link between collecting data and the development of the emergent theory that explains the data (Charmaz, 2006). Through coding, the researcher identified what was happening in the data and found what it means.

\section{RESULT AND DISCUSSION}

This present study explains how a person with SUD maintains recovery after the completion of the residential program in a drug treatment facility.

\subsection{Exhaustion Phase (Pagsadsad)}

Individuals with SUD undergo a phase wherein they experience a significant amount of distress, such 
as emotional, physical, social, spiritual, and cognitive exhaustion and fatigue. The exhaustion phase (pagsadsad) is described by the participants as:

"My life was very chaotic, disoriented, senseless, and with no purpose. I have broken relationships with my family, my parents, and my children. I became useless in the community; I became the problem of the community." (P9, 43 y.o.)

Most participants shared that this phase varies from one person to another. Some might have been rehabilitated several times and not feel exhausted about his life at all, while the other may feel like hitting rock bottom on his first attempt at recovery. Individual differences depict a vital role in this phase since every individual varies from one another in terms of personality traits, coping skills, intellectual and emotional capacity.

Likewise, this phase is characterized as feeling numb as participants described it as choosing not to feel anymore, not because they want to, but because they are exhausted about life at all. They have no other option but to breathe and wait until they die. They were all hopeless and helpless about their substance use problem and worse, they do not know what to do and who to call because they felt so isolated in the world since they became dependent on drugs.

It was dark. There was no peace of mind. I cannot feel anything, I have no emotions. I do not care about my family anymore. It came to a point that I borrowed money from everyone; family, friends, and co-workers. I even pawned my valuables just to get money to buy drugs, and worst I stole money, I joined a group wherein we robbed houses and stores. Again, things I did for drugs (laughs).” (P20, 40 y.o.)

The drugs helped them cope with their negative feelings accompanied by detachment from self, destruction of relationships, and isolation from the world hence, they strongly fear not having the drugs to cover up such ineptness and inefficiency, which resulted in becoming more vulnerable to triggers and impulses to satisfy the cravings. In a deeper sense, they are just trying to fill in the psychological emptiness they have inside such as emotional and psychological fatigue. Broken family relationships, poor level of self-esteem and worth, distorted cognition about the ability to change and to recover led to helplessness. In addition to this, negative cognitions regarding the self have been identified during this phase, wherein individuals think that everybody is against them and that they can no longer be trusted. As a result, the pain involved in such isolation is emotionally expressed either through silence, hostility, or temperamental outbursts.

"It was a disaster. Since everybody was angry at me, I hated them all. I got into a fight with my father where I attempted to stab him! I was angry too. I only did it because I was so sad during that time, I was all alone. I have no purpose in life, everyone hates me. They all wished for my death.”(P4, 58 y.o.)

Most participants have relapsed in their recovery process but were able to get on track after experiencing the Exhaustion Phase. Although they have undergone numerous confinement and treatment for SUD, they were not ready to recover during those times hence they relapsed.They shared that their drugging experience before maintaining recovery was not that significant to cause an impact and intense feelings of exhaustion that would initiate recovery, therefore, all previous confinements just resulted in numerous slips and eventual relapse. Despite being able to finish the residential program, an individual not experiencing exhaustion does not possess the initiative to start and maintain a self-directed recovery.

An individual who is undergoing the Exhaustion phase (pagsadsad), experiences strong emotional and physical fatigue. Emotionally, the individual has feelings of helplessness, withdrawal tendencies, depressive episodes, decreased level of self-worth and self-confidence, intensified anxiousness, fear of deteriorating mental state, and hopelessness. These feelings were attributed to all the negative circumstances that happened in their lives as characterized by losing a loved one, separation from family members and other significant relationships, detachment from the community, and more importantly, isolation 
from the world. Physiologically, the individual suffers from physical pain brought about by the continuous cycle of substance use. The desire to use illicit drugs never stops as the body became dependent on them. Despite careful attempts to stop, the brain structures and processes have already changed thus, seeking the pleasurable effects of the drugs remains the primary need that must be satisfied even if the physical body is no longer capable of functioning normally. Impaired control was evident marked by intense cravings and increased tolerance to the drugs (APA, 2015) hence, significant weight loss, sleeplessness, health-compromised body, perceptual disturbances, and other medical conditions are some of the consequences of substance use. The participants of this study shared that the combination of physical and emotional exhaustion is what they call hitting rock-bottom.

Despite the varying timeline and characteristics of the Exhaustion Phase, this is a subjective phenomenon that only the individual with SUD could identify within himself. It is noted that individuals may have experienced several negative events associated with substance use like being jailed, losing a significant family member, or might have developed a psychological problem yet the event was not considered and perceived by the individual as exhausting and does not trigger feelings of hopelessness, SUR will not be initiated. Nevertheless, this grounded theory discovered that the hitting the rock bottom experience was identified to be the turning point or the initiating point of SUR. The reasons for getting or staying sober were intensified negative consequences of substance use. True to the statement of Frings and Albery (2015), hitting rock bottom involves being aware of what was lost to substance use like a family relationship, purpose in life, trust, and selfrespect, and what else will be gone if they do not decide to change and recover. The exhaustion phase varies from one person to another because substance use disorder is a syndrome that manifests through several underlying dysfunctions interacting in the biopsychosocial antecedents and consequences.

\subsection{Recognition Phase (Pagkilala)}

The second phase in the process of SUR is the Recognition Phase (pagkilala) also known as the phase of gaining awareness. This is characterized as being aware of the disorder, acknowledging the existence of the substance use problem and how it is causing significant distress and dysfunction in all aspects of their lives. The participants recognized the results and consequences of their substance use thus realized their need for treatment and the importance of recovery.

"It came to the point that my parents and my siblings have me detained. I underwent detox, they coerced me into treatment, and there I started to realize the things that happened in my life. I had the drive and the realization that I am not getting any younger anymore, what has happened to me, what did the drugs do with my life? That motivated me to change." (P9, 43 y.o.)

The individual recognizes the substance use problem and the need for treatment after being exposed to the most exhausting phase of his life, the rock bottom. It is only after the exhaustion phase that the individual became aware of the disease and fully understands the essence of undergoing therapeutic interventions. When the individual subsequently recognizes the nature of knowledge as Recognition Phase (pagkilala). At this phase, the cognition of the individual improves in this phase as he learns to acknowledge that substance use has been causing dysfunctions in his life and he eventually becomes aware that he is sick and needs treatment. Realizations and recognitions of facts regarding the effects of substance use come after most exhausting circumstances in life. As discussed by Pagano, Maria, Swaringen, Shanna, and Frank (2016) individuals suffering from drug addiction are less likely to volunteer in activities related to getting sober due to a deficit in self-awareness. Therefore, the Filipino individual who has come to this phase of recovery has opened their minds and has gained awareness on the here and now, their current emotional and mental states, and their need for treatment for their disease. 


\subsection{Acceptance Phase (Pagtanggap)}

In this phase, the individual does not only acknowledge and recognize his condition, but he also accepts the reality that he is sick, and he eventually plans action on the things that he needed to do to recover. This involves accepting full responsibility for his SUR and taking steps into a lifelong drug-free.

"I made a decision, I admitted to myself that nobody can win against drugs, I cannot win against drugs. I have been in and out of different treatment facilities, I am really very tired, and therefore I realized, I need to submit myself into a program, the last time I accepted this fact, I was extremely exhausted about life." (P19, 44 y.o.)

They displayed accountability in their recovery. During this phase, support from family members was sought and the action towards maintaining SUR was initiated by the person. The Acceptance Phase (pagtanggap) takes place wherein the individual not only understands the nature of SUD but also plans on doing something about his recovery. This phase commences after the person had improved cognition and has alleviated the negative emotions and thoughts during the rock bottom experience in the Exhaustion phase. The individual has fully accepted his defeat with substance use, with planned actions, or develop strategies to combat his triggers and cravings.

\subsection{Involvement Phase (Paggalaw)}

The participants struggled to initiate SUR until they experience significant levels of exhaustion from the physical, emotional, and psychological effects of persistent drug use which subsequently resulted in gaining awareness about the disease, its consequences, and the need for treatment. Thus, the exhaustion phase progresses into recognizing and then accepting the need for treatment that leads the individual to submit to something greater than oneself and become involved in the process of treatment. The individuals surrender the things that they have no control of and become involved in activities that are associated with maintaining recovery.
With awareness of the condition, the participants likewise recognized the importance of undergoing treatment. Hence, this phase includes submitting self into a facility or program that provides interventions for drug addiction. This phase also suggests that everyone involves in the process of treatment, and they acknowledge the role of professional intervention. Being involved in the treatment program helped the participants gain control over their feelings, thoughts, and action alongside the treatment interventions coming from professionals.

"For so many years, I thought the solution to my problem was self-sufficiency, I was wrong. Hence, I started to follow the program wholeheartedly. I submitted myself to its principles. I cannot start recovering when I was outside a facility." (P8, 50 y.o.)

Involvement in the treatment process is not limited to submitting oneself to a program, the individual must open up and share his life accounts, communicate his concerns and issues, advocate for himself, participate in psychosocial interventions that address underlying trauma or other adverse childhood experiences that might have contributed to the development of the disorder. The individual must also involve himself in planning for his recovery outside the facility and be able to adhere to what has been required on his eventual discharge. The Involvement Phase (paggalaw) described the person who is seeking professional help and involves himself in a program that provides interventions for drug addiction. Upon undergoing treatment interventions, the recovering individual receives psychosocial services and interventions like spiritual enhancement, vocational and livelihood programs, and health education that taught and prepared him in the reintegration into society. The rehabilitation facility also equipped the person with skills in relapse prevention and management, and other competencies necessary for recovery. In terms of being involved in drug treatment, it is not only the responsibility of the individual with SUD to progress in this phase, but the facility or the service provider must also have competent clinicians, 
accessible psychosocial interventions that are evidence-based and deemed effective in the addiction field, and the resources to help the client take another step in the ladder of recovery which is the maintenance phase. It is also a must that the recovering individual must also participate and voluntarily involve himself in treating his unresolved issues like trauma and other deep-seated concerns to be able to address the underlying cause of his substance use disorder.

\subsection{Maintenance Phase (Pagpapanatili)}

Analysis of the data obtained generated another phase for recovery known Maintenance Phase. In this phase, the individual with SUD is maintaining a lifelong process of self-directed recovery outside the facility without returning to his previous drugrelated behaviors. According to this grounded theory, five components maintain substance use recoveries (SUR) such as autonomy, maturity, restitution, positive feelings, and support system. Autonomy gives the individual a feeling of independence, and equal access to opportunity makes the recovering person with substance use disorder sustain a selfdirected, productive, and recovery-oriented lifestyle. In addition, the maturity of an individual does not depend on the age, gender, educational attainment, and set of beliefs of a person, instead it is based on the ability to communicate, make sound decisions, and adapt to everyday circumstances in life. The participants further shared that their substance use relapse could be attributable to their cognitive, social, and emotional immaturity. Another component that maintains SUR is restitution, this study proposes that unless the person with SUD develops the need to give back or to help others, they cannot maintain recovery with their disorder. This recovery element infers believing in the capacity of the person with SUD to help others help themselves would prolong and sustain the motivation to recover. It was further identified that the support system has a great impact on combating triggers of substance use. While positive feelings maintain recovery in substance use disorder as manifested through behavioral expression such as respect, trust, acceptance, and feelings of significance in sustaining a drug-free lifestyle. Lastly, a support system like family, recovery groups and meetings, co-workers, and friends inhibit the possibility of relapsing to substance use because it instills an improved sense of belongingness which suffices the person's need for significance.

Autonomy in SUR describes a life that is governed and directed by one's thoughts, feelings, and emotions rather than by the external forces when given an equal opportunity in the community like making own decisions, pursuing a goal, performing obligations as a responsible individual in the community provided with equal access and opportunity as a normal human being, not deprived of their rights. Opportunities such as employment, education, and an equal chance to perform substantial responsibility in the family and the community make them more inclined into maintaining recovery.

To note, most of the participants are gainfully employed and with access agencies/institutions that provide inclusive education for the recovering people with SUD. In maintaining SUR, the individual must be provided with equal opportunity marked by inclusive and non-discriminatory services that they can utilize in battling with their disability. Autonomy gives the recovering person enough motivation, positive feelings, and constructive thoughts to maintain a drug-free lifestyle. As explained by Legault (2016), individuals with SUD are most likely to maintain recovery when they make things on their own, when provided with the same opportunities without discrimination in the family and community. Consistent with the study of Ryan and Deci (2010) people become engaged, happy, and interested when the need for autonomy has been sufficed. In an interdependent culture like the Philippines, autonomy is a motivational state that produces self-endorsed behaviors that concerns one's interest, values, desires, and promotes other peoples' welfare. However, there are possible barriers in acquiring autonomy such as stigma on substance users, and reluctance to seek professional help because Filipinos are generally not open to treating mental health problems. 
Maturity is operationally defined by the participants as the ability to accept, decide, communicate, and adapt in a changing environment. For them, maturity is to recover from substance use (emotional and mental maturity) with the awareness that there are substance use problems and acceptance of the need to maintain a lifelong process of recovery with the understanding that what they have is a disease, a disorder of the brain, a health issue that requires treatment from a health perspective. They are aware of the effects, relapse factors, and consequences of their behaviors.

With maturity comes an informed decision that entails a bigger responsibility. Proper communication and openness about recovery are some of the important parts of maturity. Participants acknowledged their past and were not ashamed of it. They were very open to sharing their life stories as they emphasized: the more they share, the more they are healed. However, some participants revealed that after being reintegrated into the society, some family members are manifesting negative behaviors towards them as characterized by distrust, fear, and doubts about the sincerity of their motives and their genuine drive to recover. Maturity helps in asserting positive behaviors over negative ones in terms of staying away from people who are or can be a trigger to substance use. This component in maintaining SUR also suggests that maturity is being aware of personal triggers, both internal and external barriers. Based on the study of Ryan and Deci (2010) maturity comes in three forms: responsibility, temperance, and perspective which supported the finding of this study that maturity is the ability to accept, decide, communicate, and adapt in a changing environment.

Restitution describes as a shared passion for helping others who also have become victims of substance use. Most of them work in a drug treatment facility and they wanted to do it for the rest of their lives to maintain their SUR. For some, they owned the facility, while others chose to work in the facility where they graduated. Unlike any other job, working in a rehabilitation facility like the addiction field is intrinsically rewarding as they satisfy the need to give back and maintain their recovery too.

With an enlightened self-interest, they understood that what a person does to enhance another's quality of life enhances one's quality of life too with a belief in the capacity of anyone to change and be better. A recovering person can help another person to better themselves. Likely, this means that restitution is the understanding that whatever a person does to help others also enhances one's life to a parallel degree. In maintaining SUR, the act of restitution is attributed to the thoughts and realization of the individual regarding his previous life and how he was able to get away from that life. This can be attributed to the Filipino values of "kabutihang loob" or goodness-of-will not just like any act of kindness or altruism, but it is the ability to show "kabutihang loob" to someone because they already experience it themselves most urgently manifested when someone is weak or is in need.

Support Systems including the family, optimistic friends, recovery groups, religious/spiritual groups, and community served as a vital component that maintains SUR. After graduating from a drug rehabilitation facility, all participants did not disconnect from the addiction field, they created a new set of friends from people with positive influence in their recovery, some religiously joined AA/NA groups. Moreover, when the community supports and accepts the recovering individual upon their reintegration, the need for belongingness and love is being achieved. Indeed, it was revealed that most of the participants' families were very supportive and cooperative in their journey to recovery from the external made the internal process of maintaining SUR possible.

Aside from family members, they also have a core group (i.e., friends, co-workers, and most importantly the community) which is a group of recovering people that meet at a certain time to catch up and to assist everyone in their continuing path to recovery. Another component that maintains recovery is the support system, as the recovering individual faces different challenges in their journey 
towards lifetime sobriety. Emotionally, the support system provides feeling that they do not have to limit themselves within their capacity because they have some people who support them, people who will listen, or even their mere presence of just being there, would not result in feelings of isolation that the individual felt during their drugging days. Participants who have supportive family members during the Involvement Phase (paggalaw) of recovery reported a higher level of motivation to maintain recovery. This is because Filipinos recognize that every human being although unique, is an integral part of the community. Thus, they placed a high value on regard to others, sensitivity, respect, concern, and understanding, helping out, and consideration to others' limitations. Recoveryoriented activities like meetings and consistent dialogue and communication to non-drug-related peers created pressures to make a healthy choice and sustained appropriate responses during difficult situations. Support system plays an important role in addiction recovery as they are the ones who listen and support the recovering individual. In addition to this, a strong support system has been linked to lower relapse rates. Equally, a strong support network motivates them to complete treatment and commits to continue down the road of recovery throughout their life.

Positive Feelings (eudaimonia type) among participants revealed happiness, feeling significant, being trusted, respected, supported, accepted, and loved maintains SUR. It is undeniable that there is a stigma and discrimination toward individuals recovering from SUD which contributed to difficulty and relapsing behavior. Participants confirmed one component that made their recovery possible was about having positive feelings, wherein there is room for growth and there is a sense of purpose, importance, and trust that should be earned by hard work and determination. Seemingly, participants believed in the need to maintain their recovery simply because they are being recognized and still respected in their family and community. Most of the participants accepted that they need to work hard to gain the trust of their loved ones, and it was not that easy given the fact that their families were the most affected people during their dosing years. Despite having to face such struggles of earning back their trust, after several years in recovery, the positive feelings of being significant, being trusted, and being loved were attained that resulted in maintaining their recovery.

In SUR, positive feelings include happiness, feeling significant, feeling trusted, respected, supported, accepted, and feeling loved by others. Just like the effects of illicit drugs in the brain that produces dopamine, all the positive feelings that the individual experiences likewise produce the same neurotransmitter associated with pleasures and rewards. Thus, with repeated exposure to the rewarding feelings of positive emotions, the brain is structured to ensure that we repeat life-sustaining activities or behaviors that bring pleasure to our reward system (NIDA, 2017). The increase of positive emotions, such as the sense of subjective power and freedom, greater self-esteem, and the attribution of change to internal and not to uncontrollable factors, as well as the simultaneous decrease of negative emotions, such as the sense of loss and inability resulted in the successful recovery of residential patients under a therapeutic program. Furthermore, the maintenance and continuation in addiction recovery have been associated with the increase of positive emotions like gratitude, love, sympathy, pride, serenity, and hope (Flora \& Stalikas, 2015).

\subsection{Substance Use Recovery Barriers}

Some individuals recovering from SUD tend to have relapse episodes in their recovery process despite being able to maintain recovery. Even after maintaining recovery for a long time is not a guarantee that an individual is not at risk of having a relapse. This grounded theory study revealed that Filipino individuals with SUD experience difficulties in maintaining recovery because of two recovery barriers: internal and external barriers.

For Internal Barrier, most of the participants revealed that the phenomenon of relapse is rooted in 
the individual's emotion and cognition towards self and their drug addiction recovery. Specifically, denial of the problem is the most common cause of returning to drug use among participants. Lack of awareness of the nature of the problem makes the participant susceptible and an easy target of any environmental triggers. Rationalizing drug use as a way of coping with life's difficulties is another personal barrier that hinders a person to maintain SUR. Most of the participants shared their stories of resisting relapse for a significant period but eventually giving in when the right excuse has been made to complete the rationalization process of justifying the need to use drugs.

Through rationalization and denial of the existence of the problem, recovering individuals may return to their old ways and behaviors when faced with environmental stressors. Another personal barrier under negative cognition towards self is having the wrong mindset and self-will. Participant shared, "I was also branded as a chronic relapse because I tended to do it (recovery) alone. I thought that recovery can be done through self-will." Selfwill is not enough to maintain recovery; this will only prevent substance use for a short time. Therefore, this study revealed that in addiction recovery, nobody can recover alone, they need somebody to support their recovery.

Aside from negative cognition, there are also negative emotions reported that lead to relapse. Upon reintegration, the participants enumerated their feelings during recoveries such as frustration, disappointment, extremely sad, and pressure to family members' high expectations regarding their recovery. Such anticipation of the recovery outcome from family and the community brought so much responsibility and accountability that some clients failed to cope up with.

Meanwhile, as experienced by the participants, External Barrier revealed that relapse among recovering substance users happens in the community markedly in the form of stigma and discrimination. Despite the scientific advances in the field of addiction, the community still has insufficient knowledge regarding the disease. The participants described the experience that recovering people like them are being discriminated against and stigmatized upon reintegration into society. They gave more emphasis during these times the government has declared war on drugs that immensely affect the lives of individuals trying to recover from substance use.

The external barrier such as stigmatizing drug addiction as a criminal offense leads to a decreased opportunity for recovering people to maintain sobriety outside of the rehabilitation facility. Discrimination also has a bigger effect on the recovery process because it entails less access to different services in the community such as limited employment opportunities, unjust or low salary, and neglect, physical and emotional abuse brought by their social status as recovering people with substance use disorder. In contrary to the popular belief that relapse happens around drug-using friends, the place where access to drugs is easy, on holidays and special occasions, these were only external barriers secondary to stigma and discrimination.

To maintain SUR, it is suggested that each component must be strengthened separately from one another, but they are considered interconnected in sustaining lifelong sobriety from illicit substances. While the individual has a strong maintenance component like a support system but lacks autonomy and experiences discrimination and rejection from the community, the desired recovery outcome can be hampered that might lead to substance use relapse if not addressed appropriately. Thus, this grounded theory strongly proposes that the individual recovering from SUD must be aware of his recovery maintenance components to be able to identify areas of their lives that need to be reinforced and supported.

Consequently, some barriers hinder an individual to maintain SUR after the cessation of their treatment program in a facility, these are internal barriers and external barriers. The external barriers make the individual reluctant to seek employment or to avail of other psychosocial services due to stigma from the community. This also results in avoiding situations that will eventually lead to devaluation and 
discrimination of their abilities. One principle of recovery stated that recovery has cultural dimensions, thus the individual's cultural experiences would greatly affect and shape the person's recovery path. This study suggested that the individual recovering from substance use disorder must be able to strive and fight the stigma in the community by confronting and accepting them as a part of their process in recovery. According to a study written by Wahl (2012), the stigma associated with addiction was also considered a strong recovery barrier that impedes recovery maintenance. Whereas the internal barriers include denial of the problem and rationalizing reasons for substance use coupled with negative feelings marked by disappointment, sadness, and failing the expectations of the family and the community. Further results showed that personal barriers such as negative emotions and mindset hinder addiction recovery (Duffy \& Baldwin, 2013), and the most frequent barrier to substance recovery was the denial of the problem hence individuals treat the disease as not serious enough (McQuaid, Jesseman, Rush, 2018). This study suggested that internal barriers could be overcome by the participants' abilities to motivate themselves, persevere in the face of frustrations, ability to regulate moods, emotions, and behaviors in maintaining a lifelong process of recovery

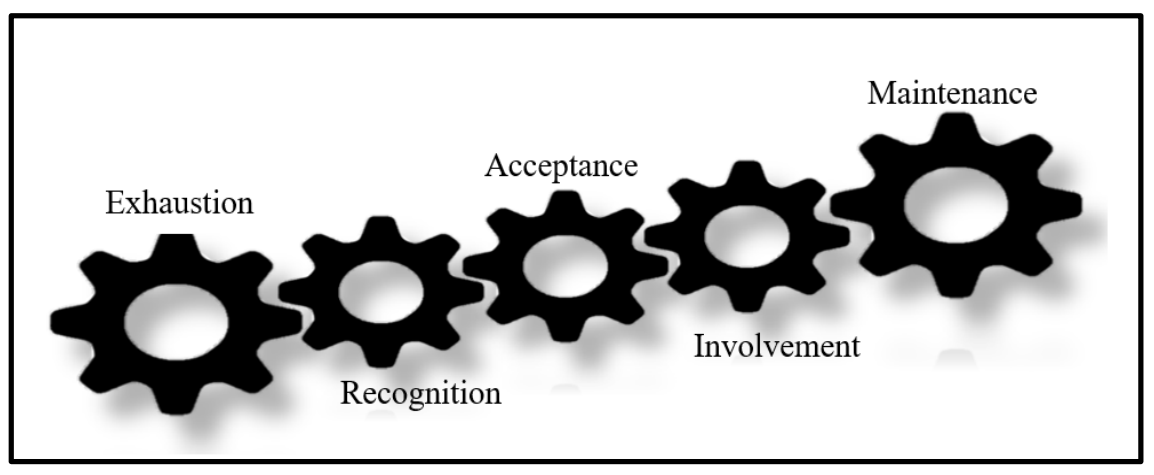

Figure 1. Theory of Filipino Substance Use Recovery (SUR)

As a product, a newly generated Theory of Filipino Substance Use Recovery (SUR) comprises five (5) different phases that include the exhaustion phase, recognition phase, acceptance phase, involvement phase, and maintenance phase. This study had also identified sub-categories that demonstrate the components in maintaining recovery from substance use such as autonomy, maturity, restitution, support system, and positive feelings. On the other hand, recovery barriers were also identified as factors contributing to substance use relapse.

As limitations, this grounded theory study developed a theoretical framework that explained the phases that a Filipino undergoes in SUR across cultures and societies. This study acknowledged several issues that need to be considered and warrant:
(1) the experiences shared by the participants are only of those who have undergone a residential treatment program and limits the information from those recovering individuals with SUD who did not undergo inpatient treatment and rehabilitation or those who have attended an outpatient program or those who did not enter any treatment facility at all; (2) the participants are heterogeneous in terms of their confinement history since half of the participants are first-timers and the other half have been admitted to a different rehabilitation facility several times before they maintain their recovery up to present; and (3) the results cannot be generalized to the population of women because a homogenous sampling is recommended as men and women may have different experiences in the SUR. 
Indeed, SUD is a chronic relapsing brain disease that requires a lifetime recovery. Scientific evidence revealed that an individual does not recover from addiction, they only manage and maintain it like any other chronic medical problems such as hypertension and diabetes, it was proven that they can return to their everyday functions and live in a community like other people through a sustainable, selfdirected, and recovery-oriented lifestyle. Therefore, the results of this grounded theory study, specifically the theory of Filipino SUR can be useful to the growing and evolving body of knowledge in the addiction field in terms of prevention, harm reduction, intervention, and recovery. This framework also provided the underpinning of how the government should address the current issues of substance use in a developing country like the Philippines by establishing treatment programs and interventions grounded and based on a theory that is developed and contextualized locally. Addiction professionals could also benefit from this study as they can develop new instrumentation and tools tailored to the needs of individuals with SUD in the country.

\section{CONCLUSION}

A theoretical foundation on how a Filipino individual recovers from SUD served as the contribution of this study. This theory suggests a process of SUR requiring an individual to undergo five phases: exhaustion (pagsadsad), recognition (pagkilala), acceptance (pagtanggap), involvement (paggalaw), and maintenance (pagpapanatili). Once the recovery started through the initial phase, individuals could maintain a drug-free lifestyle when provided with these recovery maintenance components: autonomy, which embraces equal opportunity, responsibility, and accessibility without stigma and discrimination; maturity, that understands and acknowledges the nature of the disease, and the ability to communicate and adapt to the changing environment; restitution, that the individual does things to enhance the lives of others which gives the same degree of fulfillment to himself; support system, suggests that support from others motivates the individual to sustain sobriety; lastly, when showered with positive feelings characterized by feeling significant, being trusted and respected by their family and the community, individual increases level of their self-worth and self-efficacy to maintain SUR. Based on the findings grounded from data, the proponents generated a theory on addiction recovery among individuals with SUD in the Philippines.

\section{REFERENCE}

American Psychiatric Association [APA]. (2015). Diagnostic and statistical manual of mental disorders (DSM-5). Arlington: American Psychiatric Publishing.

Charmaz, K. (2006). Constructing Grounded Theory: A Practical Guide Through Qualitative Analysis. London: SAGE Publication.

Duffy, P., Baldwin, H. (2013). Recovery posttreatment: plans, barriers, and motivators. Substance abuse treatment, prevention, and policy, 8(6), 101-186.

Fitzgerald, H.E. \& Puttler, L. I. (2018). Alcohol use disorders: A developmental science approach to etiology. New York: Oxford University Press.

Flora, K. \& Stalikas, A. (2015). Positive emotions and addiction. International Journal of Psychosocial Rehabilitation, 19(1), 57-85.

Frings, D. \& Albery, I. P. (2015). The social identity model of cessation maintenance: formulation and initial evidence. Addict Behavior, 44, 35-42.

Goldstein, R. Z. \& Volkow (2011). Dysfunction of the prefrontal cortex in addiction: neuroimaging findings and clinical implications. Nat Rev Neuroscience, 12(11), 652-669.

Hser, Y. I., Evans, E., Grella, C., Ling, W., \& Anglin, D. (2015). Long-term course of opioid addiction. Harvard review of psychiatry, 23(2), 76-89.

Kleber, H. D., Weiss, R. D., Anton, R.F., George, T.P., Greenfield, S.F. (2011). Treatment of patients with substance use disorders, second edition. American Psychiatric Association, 164, 5-123. 
Laudet, A. (2013). Life in recovery: Report on the survey findings. Washington: Faces \& Voices of Recovery.

Legault, L. (2016). The Need for autonomy. Encyclopedia of personality and individual differences.

McQuaid, R. J., Jesseman, R., \& Rush, B. (2018). Examining barriers as risk factors for relapse: A focus on the Canadian treatment and recovery system of care. The Canadian journal of addiction, 9(3), 5-12.

McQuaid, R.J., Malik, A., Moussouni, K., Baydack, N., Stargardter, M., \& Morrisey, M. (2017). Life in Recovery from Addiction in Canada. Ottawa: Canadian Centre on Substance Use and Addiction.

Moeller, S. J., \& Goldstein, R. Z. (2014). Impaired self-awareness in human addiction: Deficient attribution of personal relevance.Trends in cognitive sciences, 18(12), 635-641.

National Institute on Drug Abuse [NIDA]. (2017). National Institutes for Health. Retrieved from http://drugabuse.gov:

https://www.drugabuse.gov/

Pagano, M. E., Swaringen, S. E., \& Frank, S. H. (2016). Low other-regard and adolescent addiction. Journal of Child \& Adolescent Substance Abuse, 1.

Ramalho, R., Adams, P., Huggard, P., \& Hoare,. (2015). Literature review and constructivist grounded theory methodology. Qualitative Social Research, 16(3).

Ryan, R. M., Deci, E. L. (2010). Self-determination theory and the facilitation of intrinsic motivation, social development, and wellbeing. Am. Psychol., 55, 68-78.
Scott, C. K., \& Dennis, M. L. (2012). The first 90 days following release from jail: Findings from the recovery management checkups for women offenders (RMCWO) experiment. Drug and Alcohol Dependence, 125(2), 110-118.

Scott, C. K., Dennis, M. L., Laudet, A., Funk, R. R., \& Simeone, R. S. (2011). Surviving drug addiction: The effect of treatment and abstinence on mortality.

Sheedy, C. K., \& Whitter, M. (2013). Guiding principles and elements of recovery-oriented systems of care: What do we know from the research? HHS Publication, 09-4439.

Substance Abuse and Mental Health Services Administration [SAMHSA]. (2018). Mental and Substance Use Disorders. https://www.samhsa.gov/ disorders. Published June 20, 2014. Accessed June 4, 2018.

Wahl, O. F. (2012). Stigma as a barrier to recovery from mental illness. Trends in Cognitive Sciences, 16(1), 9-10.

Whitesell, M., Bachand, A., Peel, J., Brown, M.. (2013). Familial, social, and individual factors contributing to risk for adolescent substance use. Journal of Addiction.

Winters, K. C., Botzet, A. M., \& Fahnhorst, T. (2011). Advances in adolescent substance abuse treatment. Current Psychiatry Reports, $13,416-421$.

Witkiewitz, K. (2011). Predictors of heavy drinking during and following treatment. Psychology of Addictive Behaviors, 25(3), 426.

Article submitted : 7 April 2021

Article accepted : 22 October 2021 